\title{
Multiple extrafoveal macular holes following internal limiting membrane peeling
}

This article was published in the following Dove Press journal: International Medical Case Reports Journal

\section{Nazimul Hussain \\ Sandip Mitra}

Department of Ophthalmology, Al Zahra Hospital, Sharjah, United Arab Emirates
Correspondence: Nazimul Hussain Department of Ophthalmology, Al Zahra Hospital, PO Box: 3499, Sharjah, United Arab Emirates

Tel +97| 65168888

Fax +97। 65637269

Email nazimul.hussain@gmail.com
Objective: Internal limiting membrane (ILM) peeling has been the standard of treatment for macular holes. Besides, causing retinal nerve fiber layer surface abnormality, postoperative extrafoveal multiple retinal holes is a rare phenomenon following ILM peeling. We report an unusual complication of eight extrafoveal macular holes occurring following ILM peeling.

Case presentation: A 60-year-old male presented with complaints of decreased and distorted vision in the right eye. He was diagnosed as having epiretinal membrane with lamellar macular hole. He underwent $23 \mathrm{G}$ pars plana vitrectomy, brilliant blue assisted ILM peeling and fluid gas exchange. Intraoperatively, ILM was found to be adherent to the underlying neurosensory retina. One month after cataract surgery, he underwent YAG capsulotomy in the right eye. $\mathrm{He}$ complained of visual distortion. His fundus evaluation in the right eye showed multiple (eight) extrafoveal retinal holes temporal to the macula clustered together.

Conclusion: This case demonstrated that peeling of ILM, especially when it is adherent to the underlying neurosensory retina, may cause unwanted mechanical trauma to the inner retina. Glial apoptosis and neuronal degeneration may presumably play a role in delayed appearance of multiple (eight) extrafoveal macular holes, which has not been reported earlier.

Keywords: internal limiting membrane, lamellar macular hole, full thickness macular holes, epiretinal membrane

\section{Introduction}

Internal limiting membrane (ILM) peeling has become an integral part of successful macular hole and epiretinal membrane surgery. Advancement in small gauge disposable forceps and membrane instruments to peel the ILM has also made surgical maneuver easy and confident. Eccentric macular hole or paramacular hole following either membrane peeling or ILM peeling for macular pathology has been reported. ${ }^{1-10}$

We describe a unique case of lamellar macular hole, which underwent ILM peeling using disposable ILM forceps and developed eight extrafoveal macular holes noticed in the postoperative period.

\section{Case presentation}

A 60-year-old male presented with complaints of decreased and distorted vision in the right eye. He gave history of undergoing macular hole surgery with phacoemulsification and intraocular lens implantation elsewhere in the left eye 5 years ago. He is hypertensive on treatment. 
On examination, his best-corrected visual acuity was $6 / 18$ in both eyes. Anterior segment examination was unremarkable in both eyes, except that the lens showed grade 2 nuclear sclerosis in the right eye and posterior chamber intraocular lens was in place in the left eye. Intraocular pressure was 16 and $17 \mathrm{mmHg}$ in the right and left eye, respectively. Dilated fundus examination showed clear ocular media and attached retina in both eyes. Right eye macula showed epiretinal membrane with macular hole, and left eye macula showed retinal pigment epithelium atrophy and scar affecting the fovea partially. Optical coherence tomography (OCT; Topcon 3D OCT 200 series; Topcon Corporation, Tokyo, Japan) showed epiretinal membrane with thin neurosensory retinal tissue at the base of the macular hole in the right eye (Figure 1). The left eye showed foveal thinning. Based on the clinical findings, a diagnosis of epiretinal membrane with lamellar macular hole and closed macular hole with foveal thinning was made in the right eye and the left eye, respectively.

He underwent $23 \mathrm{G}$ pars plana vitrectomy, brilliant blue (BBG)-assisted ILM peeling and fluid gas exchange in the right eye. ILM was peeled with pinch and peel technique using disposable $23 \mathrm{G}$ ILM forceps. BBG was used for the second time to stain the ILM in the central area of macula where the epiretinal membrane was present and ILM was completely peeled. ILM was found to be adherent to the underlying neurosensory retina. Postoperative period was uneventful. At 1-month follow-up, his visual acuity was $6 / 18$ in the right eye. However, there was evidence of Grade 2 nuclear sclerosis with posterior subcapsular cataract in the right eye. OCT showed closed macular hole, and fundus

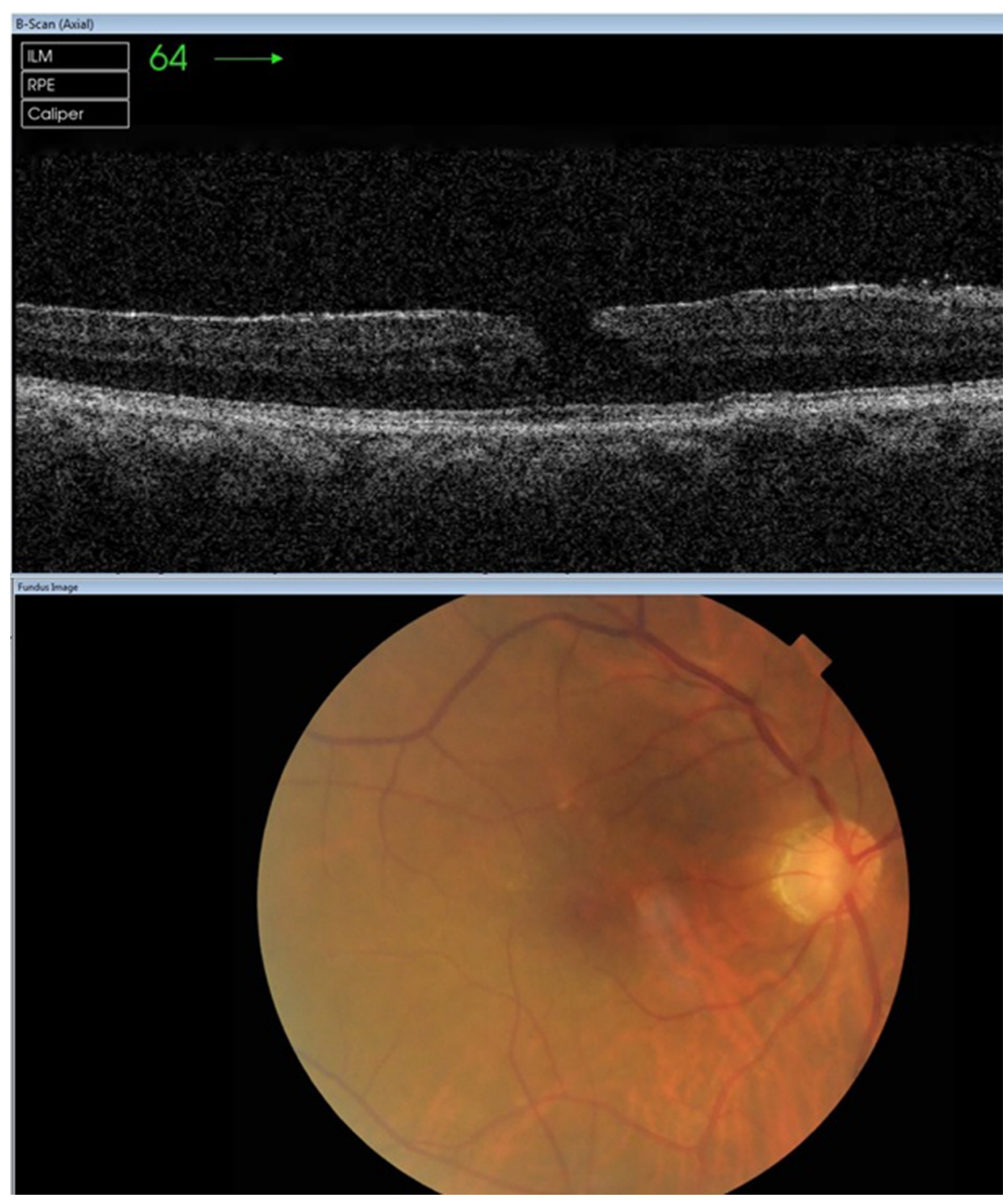

Figure I OCT image of lamellar macular hole with epiretinal membrane (top). Bottom shows corresponding color fundus image. Abbreviations: ILM, internal limiting membrane; OCT, optical coherence tomography; RPE, retinal pigment epithelium. 


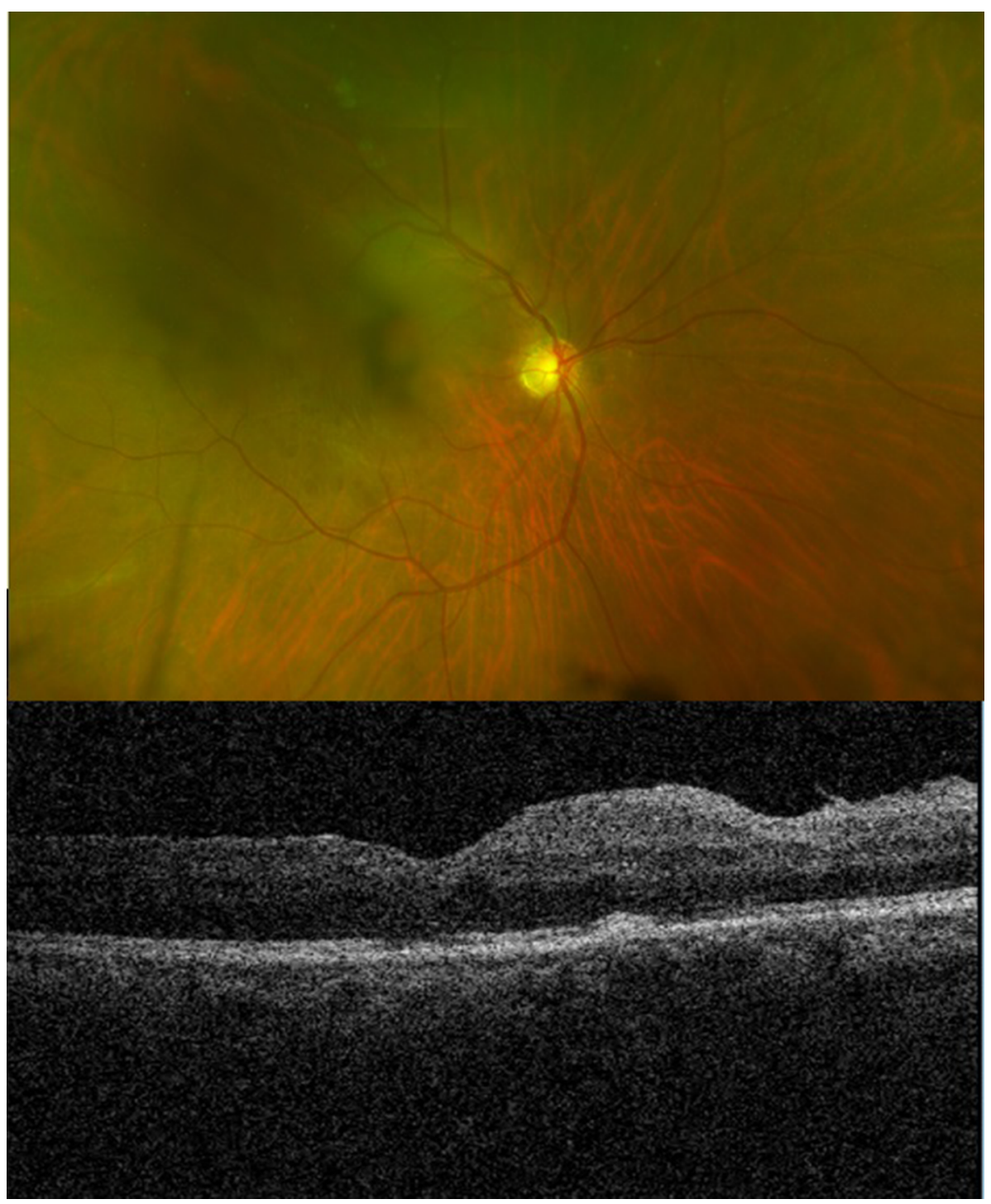

Figure 2 Postoperative fundus color image showing a central haze due to early cataract (top) and OCT image showing maintained foveal contour (bottom). Abbreviation: OCT, optical coherence tomography.

picture showed central hazy view due to the presence of cataract (Figure 2).

Three months later, his visual acuity in the right eye progressively worsened and he underwent phacoemulsification with intraocular lens implantation in the right eye. One month after cataract surgery, he underwent YAG capsulotomy for posterior capsular opacification in the right eye. He complained of visual distortion. His fundus evaluation in the right eye showed clear ocular media, attached retina and closed macular hole, but multiple extrafoveal retinal holes were seen temporal to the macula which were clustered together. Eight retinal holes were noticed (Optos 200Tx, UWF ${ }^{\mathrm{TM}}$; Optos Plc, Dunferline, UK; Figure 3). Serial OCT scan was used, so that all the possible axial scans ran through all the holes possible within the scan. It showed evidence of extrafoveal full-thickness holes with neurosensory intraretinal cystic changes extending toward the parafoveal area associated with minimal subretinal fluid (Figure 4). He underwent immediate barrage laser photocoagulation in the right eye (Figure 5A). On regular follow-up, his ocular condition stabilized and he informed that his visual distortion had decreased. When reviewed at the end of 6 months, his best-corrected visual acuity improved to $6 / 9$ in the right eye. Fundus examination showed well-barraged laser marks around the holes (Figure 5B). OCT showed closed macular hole and flattened retina temporal to the fovea.

\section{Discussion}

Postoperative extrafoveal multiple retinal holes is a rare phenomenon occurring after ILM peeling. Most of these 


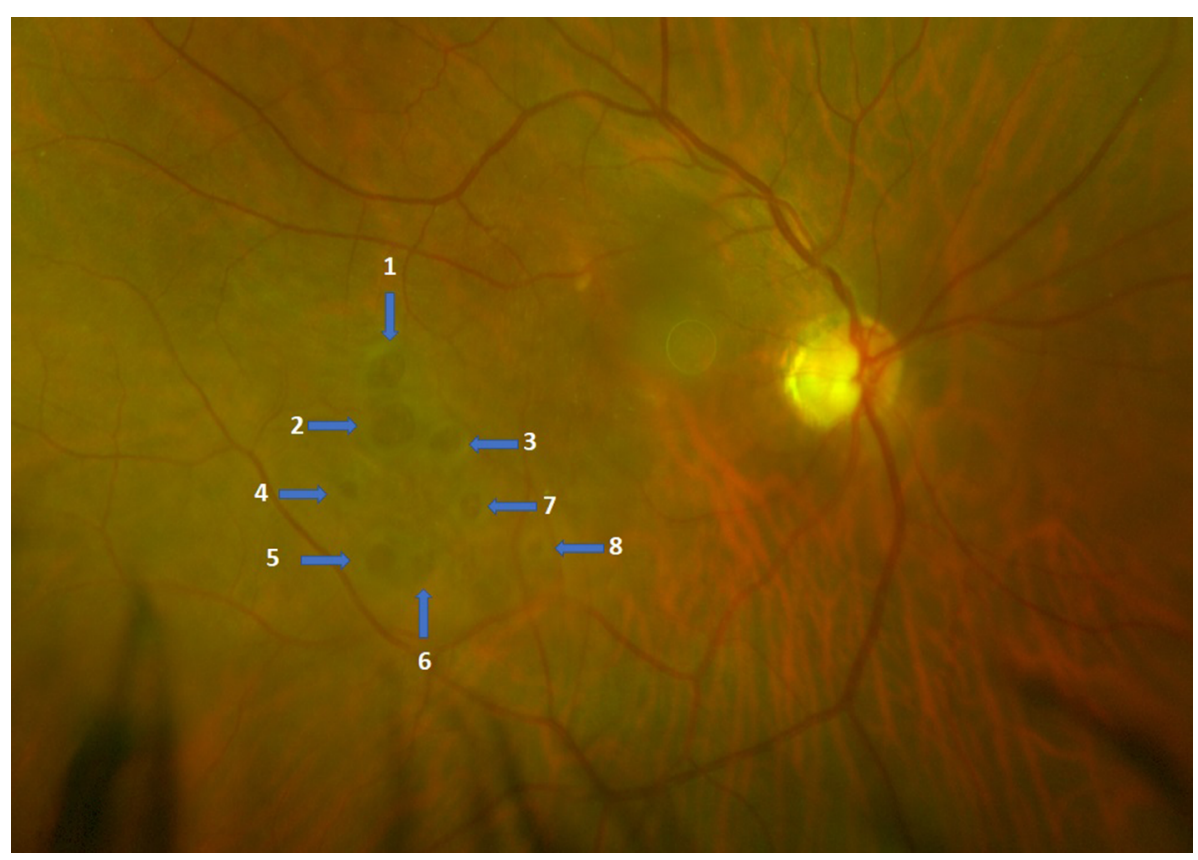

Figure 3 Color fundus image showing eight extrafoveal retinal holes (arrows/numbered).

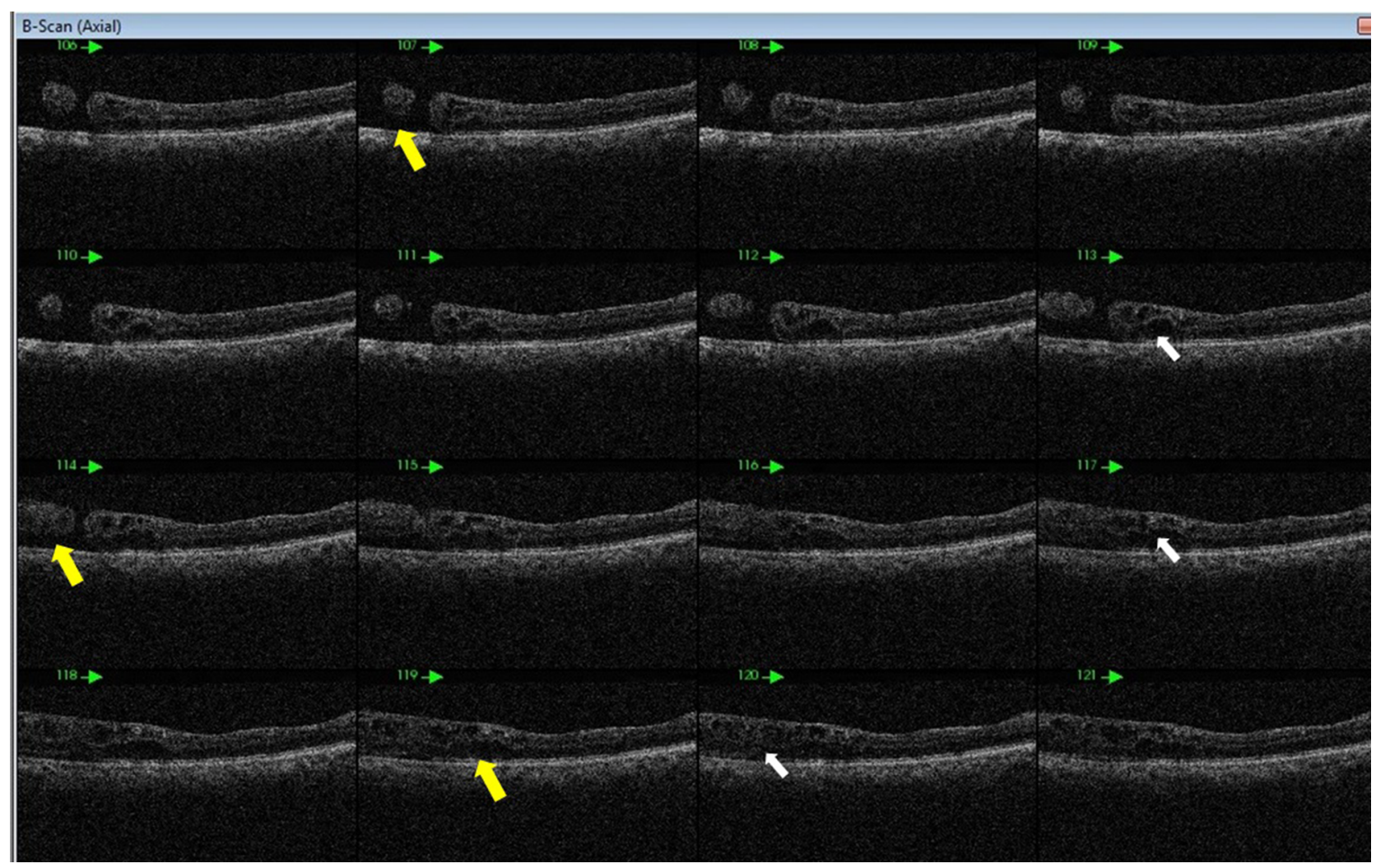

Figure 4 Serial OCT axial scans showing subretinal fluid (yellow arrows) in the area of retinal holes and neurosensory retina cystic changes (white arrows). Abbreviation: OCT, optical coherence tomography. 


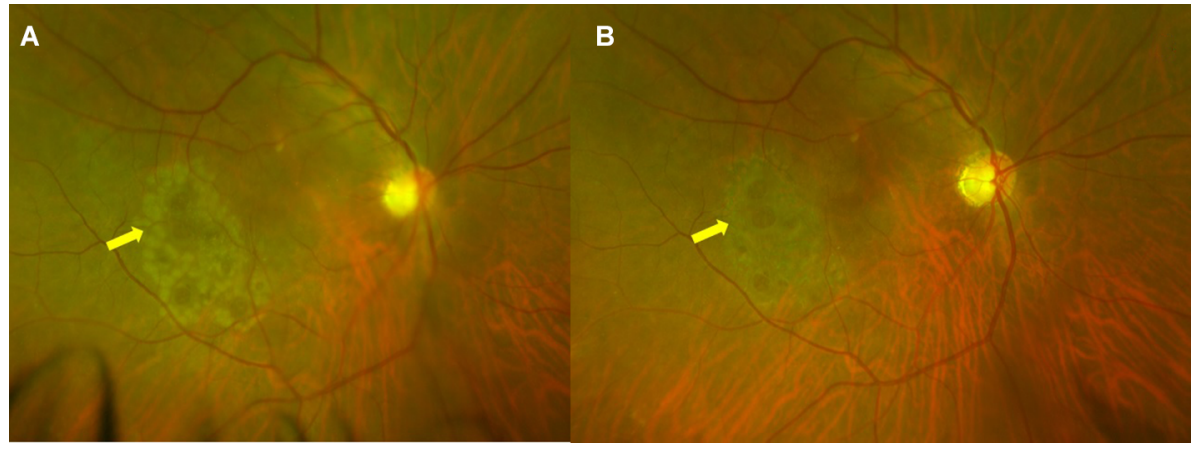

Figure 5 Post laser fundus image.

Notes: (A) Color fundus image showing fresh barrage retinal photocoagulation marks (arrow); (B) shows barrage retinal photocoagulation marks I month later (arrow).

macular holes were described to happen between 1 week and 3 years. ${ }^{1-8}$ Iatrogenic full-thickness macular holes following vitrectomy for ILM peeling for idiopathic macular hole was first reported by Rubinstein et al. ${ }^{7}$ The authors retrospectively reviewed 232 consecutive patients who underwent ILM peeling during macular hole surgery over a period of 5 years. Four patients were found to have developed eccentric macular holes following surgery, which were noticed between 3 and 6 months. The holes were located between 4 o' clock and 6 o' clock position in three patients and at 9 o' clock position in one patient.

Yeh et $\mathrm{al}^{5}$ have reported a case of full-thickness macular hole below the superotemporal arcade without any associated subretinal fluid noticed 2 months after epiretinal membrane surgery. Steven et $\mathrm{al}^{9}$ also described seven patients with temporal paracentral macular holes after ILM delamination for different macular pathologies. Mason et $\mathrm{al}^{6}$ reported development of eccentric macular hole after ILM peeling for epimacular proliferation (macular pucker). The eccentric macular holes were noticed at a mean of 3.1 months. Of these six eccentric macular holes, four were located temporal to the fovea, one was located superior to the fovea and one was located nasal to the fovea. None of the patients underwent any treatment, as the patients were asymptomatic and OCT showed full-thickness and flat macular holes.

Abo El Enin et $\mathrm{al}^{4}$ described six eyes that developed eccentric macular hole after surgery for idiopathic epimacular proliferation. The authors noted four eccentric macular holes in the temporal, one in the superior and one nasal to fovea. None of the holes increased in size or caused visual symptoms and, hence, were left as such without any complications.

Sandali et $\mathrm{al}^{1}$ and Rush et $\mathrm{al}^{3}$ in their retrospective review of patients after macular surgery found the incidence of paracentral and eccentric macular holes as $0.66 \%$ and
$2.1 \%$, respectively. Most of the published literature suggests occurrence of single eccentric retinal holes, but Brouzas et $\mathrm{al}^{2}$ found the incidence of postoperative eccentric macular holes to be $2.5 \%$ in their retrospective case series study. This case series showed that all cases developed single eccentric macular holes except two cases which developed multiple holes. One patient developed two and the other four eccentric macular holes.

The characteristic of the case presented here is development of multiple extrafoveal macular holes noticed 3 months postoperatively. It is notable that eight extrafoveal full-thickness macular holes developed, and all of them were noticed in the temporal quadrant. There was visual distortion associated with intraretinal cystic change and minimal subretinal fluid as seen on OCT, hence the indication for barrage laser photocoagulation. Presence of eight extrafoveal macular holes after ILM peeling has never been reported earlier.

Rubinstein et $\mathrm{al}^{7}$ first reported iatrogenic macular holes following ILM peeling for idiopathic macular holes. The authors believed that formation and location of these iatrogenic macular holes represents trauma to the underlying layers of the retina. This may occur during grasping of the ILM with forceps either at the initial site of grasping or subsequent regrasping of the membrane. It was believed that holes were not obvious at the time of surgery, although small areas of hemorrhage, which develop during peeling, may possibly indicate the site of subsequent hole formation.

Studies $^{1-3,6,7}$ also highlight that the use of BBG or indocyanine green dye is not the primary cause of iatrogenic hole formation, even though indocyanine green dye is known to cause retinal pigment epithelium or photoreceptor toxicity and weakening of glial structure of the retina by causing decapitation of Müller cells at all instances. Mechanical trauma to the retinal nerve fiber layers appears to be the 
primary cause of paracentral macular holes following ILM peeling. Several theories have been implicated, and each reported incidence suggests multiple other factors besides mechanical trauma during peeling of ILM. Sandali et al ${ }^{1}$ suggested that paracentral macular holes occurred at the limits of ILM peeling. The contracture of the edge of peeled ILM has been suggested as the cause of eccentric macular hole formation. It has been suggested that tangential ILM edge contracture may explain the delayed occurrence of these holes and their location at the edge of the ILM peeling.

Brouzas et $\mathrm{al}^{2}$ suggested that pathogenesis of macular holes was consistent with the contraction of residual ILM or secondary epimacular proliferation stimulated by ILM peeling. Peeling of ILM can possibly traumatize underlying muller cells, which may be enough to cause secondary delayed degeneration of adjacent retinal neuronal cell structures. ${ }^{11}$ During such removal, ILM necrosis occurs, which may result in glial apoptosis and diminished retinal function due to absence of inner foot plates of muller cells. ${ }^{12}$ Rush et $\mathrm{al}{ }^{3}$ believed that eccentric macular holes in their study is most consistent with contraction of the remaining edge of ILM or induced shear stress along the margin of the ILM either at or after the time of peeling, resulting in the expansion of a previously undetectable retinal defect.

In our single case with eight multiple extrafoveal holes, the location of the holes were in the temporal quadrant clustered together (Figure 3). While reviewing the surgical video, we observed that the ILM peeling was initiated in the inferotemporal area outside the area of epiretinal membrane (area where BBG color was seen). During the pinch and peel, the ILM was found to be adherent to the underlying retina, and hence, there was irregular peel initially in the particular quadrant with multiple times of attempt to pinch and peel the ILM. Hence, multiple focal areas of hemorrhage and retinal opacification were noticed, suggesting mechanical trauma (Figure 6). Delayed occurrence after 3 months probably could suggest delayed neuronal degeneration and apoptosis of glial cells, besides mechanical trauma. Hence, a combined mechanism may have resulted in this unwanted situation.

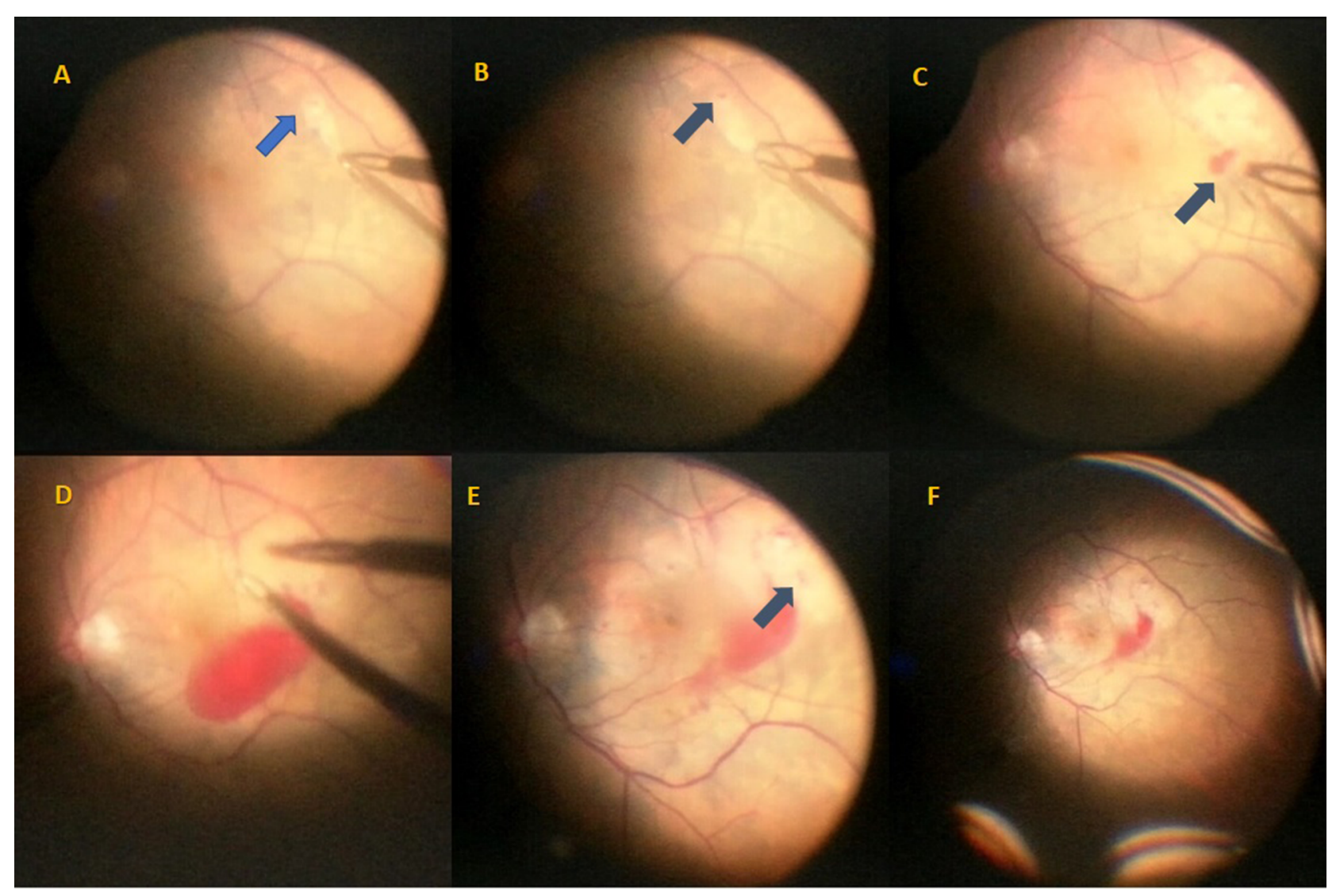

Figure 6 Intraoperative images during ILM peeling.

Notes: (A) Intraoperative fundus images during ILM peeling showing the initial site of pinch and peel (arrow); (B) focal retinal hemorrhage (arrow); (C and D) other areas of retinal hemorrhage (arrow); (E) whitening of the retinal surface (arrow); and (F) initiation of fluid air exchange. Abbreviation: ILM, internal limiting membrane. 


\section{Conclusion}

Our case demonstrated that peeling of ILM, especially when it is adherent to underlying neurosensory retina, may cause unwanted trauma to the inner retina, resulting in multiple extrafoveal macular holes.

\section{Acknowledgments}

Written informed consent was provided by the patient to have the case details and any accompanying images published. Approval of the Al Zahra Hospital Ethics committee was sought and obtained for the preparation of manuscript and publishing it.

\section{Disclosure}

The authors report no conflicts of interest in this work.

\section{References}

1. Sandali O, El Sanharawi M, Basli E, et al. Paracentral retinal holesoccurring after macular surgery: incidence, clinical features and evolution. Graefes Arch Clin Exp Ophthalmol. 2012;250(8):1137-1142.

2. Brouzas D, Dettoraki M, Lavaris A, Kourvetaris D, Nomikarios N, Moschos MM. Postoperative eccentric macular holes after vitrectomy and internal limiting membrane peeling. Int Ophthalmol. 2017;37(3):643-648.
3. Rush RB, Simunovic MP, Aragon AV, Ysasaga JE. Postoperative macular hole formation with internal limiting membrane peeling for the treatment of epiretinal membrane. Retina. 2014;34(5):890-896.

4. Abo El Enin MA, El Tourky HM, Swelam A. Non-foveal macular holes after PPV for macular pucker. Middle East Afr J Ophthalmol. 2010;17(3):254-256.

5. Yeh S, Bourgeois KA, Benz MS. Full thickness macular hole following vitrectomy with internal limiting membrane peeling. Ophthalmic Surg Lasers Imaging. 2007;38(1):59-60.

6. Mason JO, Feist RM, Albert MA Jr. Eccentric macular holes after vitrectomy with peeling of epimacular proliferation. Retina. 2007;27(1): $45-48$.

7. Rubinstein A, Bates R, Benjamin L, Shaikh A. Iatrogenic eccentric full thickness macular holes following vitrectomy with ILM peeling for idiopathic macular holes. Eye (lond). 2005;19(12):1333-1335.

8. Chatziralli I, Theodossiadis G, Douvali M, Rouvas AA, Theodossiadis P. Eccentric macular hole after pars plana vitrectomy for idiopathic macular hole: a case report. Case Rep Ophthalmol. 2017;8(1): 116-119.

9. Steven P, Laqua H, Wong D, Hoerauf H. Secondary paracentral retinal holes following internal limiting membrane removal. Br J Ophthalmol. 2006;90(3):293-295.

10. Kozak I, Freeman WR. Nonprogressive extrafoveal retinal hole after foveal epiretinal membrane removal. Am J Ophthalmol. 2006; 141(4):769-771.

11. Wolf S, Schnurbusch U, Wiedemann P, Grosche J, Reichenbach A, Wolburg H. Peeling of the basal membrane in the human retina: ultrastructural effects. Ophthalmology. 2004;111(2):238-243.

12. Newman E, Reichenbach A. The muller cell: a functional element of the retina. Trends Neurosci. 1996;19(8):307-312.
International Medical Case Reports Journal

\section{Publish your work in this journal}

The International Medical Case Reports Journal is an international, peer-reviewed open-access journal publishing original case reports from all medical specialties. Previously unpublished medical posters are also accepted relating to any area of clinical or preclinical science. Submissions should not normally exceed 2,000 words or

\section{Dovepress}

4 published pages including figures, diagrams and references. The manuscript management system is completely online and includes a very quick and fair peer-review system, which is all easy to use. Visit $\mathrm{http}: / /$ www.dovepress.com/testimonials.php to read real quotes from published authors. 